\title{
Closed-loop Kinesthetic Stimulation for the Treatment of Sleep Apnea Syndromes
}

\author{
D. Pérez ${ }^{1,2}$, G. Guerrero ${ }^{1,2}$, D. Feuerstein ${ }^{3}$, L. Graindorge ${ }^{3}$, A. Amblard ${ }^{3}$, J-L. Pépin ${ }^{4}$, L. Senhadji ${ }^{1,2}$, \\ A. I. Hernández ${ }^{1,2}$ \\ ${ }^{1}$ INSERM, U1099, Rennes, F-35000, France \\ ${ }^{2}$ Université de Rennes 1, LTSI, Rennes, F-35000, France \\ ${ }^{3}$ Sorin CRM SAS (a LivaNova company), Clamart, France \\ ${ }^{4}$ Univ Grenoble Alpes, CHU Grenoble, Sect Physiol Sommeil \& Exercice, Inserm U1042, Pole \\ Thorax \& Vaisseaux, Lab EFCR, F-38000 Grenoble, France
}

\begin{abstract}
Sleep apnea syndrome (SAS) is a common disease characterized by recurrent episodes of breathing pauses or important reductions in respiratory amplitude during patient's sleep. These episodes provoke significant cardiorespiratory modifications that may have long-term cardiovascular consequences. This paper proposes a novel approach for the treatment of SAS, based on a closedloop kinesthetic stimulation, adapted as a function of the patient's physiological response. The closed-loop control system is based on concurrent, coupled proportionalderivative $(P D)$ controllers that modulate the stimulation amplitude delivered to the patient. The controller was tested on a first phase of a clinical protocol including 12 patients with previously diagnosed SAS. Results from one patient are presented in this paper, showing how the proposed controller is capable to adapt stimulation parameters, converging to a minimum patient-specific amplitude. These results are encouraging regarding the feasibility of the integration of the proposed controller into the therapy for the adaptive optimization of the kinesthetic stimulation amplitude and warrant a second phase of the clinical study of this system.
\end{abstract}

\section{Introduction}

Sleep apnea syndrome (SAS) is a common disease characterized by recurrent episodes of breathing pauses (apnea) or important reductions in respiratory amplitude (hypopnea) during patient's sleep. Although this syndrome affects more than $5 \%$ of the general population [1], it remains under-diagnosed. SAS provoke an alteration of the sleep structure (sleep fragmentation), as well as acute cardiorespiratory responses, that have been associated with chronic pathologies such as hypertension and certain metabolic disorders [2][3]. The current recommended treatment for patients suffering SAS is the continuous positive airway pressure (CPAP) therapy [4]. However, patient compliance to this therapy is low, with an average adherence rate between $39 \%$ and $50 \%$ [5].

We have recently proposed a novel system (PASITHEA) for real-time monitoring and treatment of SAS, based on triggered kinesthetic stimulation. This system triggers a mechanical stimulation applied to the skin of the patient when apnea or hypopnea events are detected by an automatic, real-time respiratory detector. The system is composed of three elements: i) a modified cardiorespiratory ambulatory recorder (Holter) based on the commercially available Sorin Holter system (SORIN CRM, Clamart, France), for real-time acquisition, recording and wireless transmission of two electrocardiogram (ECG) channels, nasal pressure (NP) and blood oxygen saturation $(\mathrm{SaO} 2)$ during a whole night; ii) a kinesthetic stimulation system (LTSI INSERM U1099, Rennes, France) and iii) a realtime control application for adaptive kinesthetic stimulation, running in a standard computer. These elements communicate through a wireless communication protocol.

A complete description of the original PASITHEA system, along with preliminary results from its first clinical evaluation has been recently published [6]. This first evaluation was based on an "on-off" control algorithm, which triggers the kinesthetic stimulation as a function of respiratory event detections. Also, the stimulation parameters (amplitude, burst duration, silent interval, maximum number of bursts) were empirically fixed for all patients. Results showed that the patients who responded to therapy presented a reduced duration of respiratory events and an attenuation of the consequent hypoxia events and acute cardiorespiratory responses during the periods in which the therapy was active. However, not all patients responded correctly to the therapy. We hypothesize that these non- 
responders may be minimized with the integration of a closed-loop control, capable to adapt and personalize stimulation parameters. In this work, we propose the integration of such a real-time closed-loop control method, as an improvement of the original system. Preliminary results based on data acquired from the first phase of a clinical evaluation protocol are also presented.

\section{Method}

\subsection{Controller description}

A closed-loop control system, integrating concurrent, coupled proportional-derivative (PD) controllers is proposed in this work to manage the kinesthetic stimulation amplitude delivered to the patient by the therapeutic system. The control system is composed of three main modules operating in real-time: i) a signal processing module, ii) control activation and iii) the coupled PD controller.

The signal processing module (Fig. 1) performs data processing on input signals which are: i) the NP signal, ii) one ECG lead and iii) the $\mathrm{SaO} 2$ signal. QRS detection is performed on the ECG signal and the instantaneous heart rate (HR), as well as the $\Delta \mathrm{HR}$ signal are derived. The $\mathrm{SaO} 2$ signal is delayed and low-pass filtered (FSaO2), to then calculate its derivative. This pre-processing is applied in order to compensate for the fact that the consequences of respiratory events on the $\mathrm{SaO} 2$ signal are observed after a certain physiological delay. The NP signal is processed by a real-time apnea/hypopnea detector which has three possibles outputs: no apnea/hypopnea detected, apnea detected and hypopnea detected. A complete description of the apnea/hypopnea detector was published in [7].

The control activation module enables the coupled PD controller module when it receives as input an apnea or hypopnea detection and will disable it in case of a return to normal respiration. Five control variables are presented as input to the coupled PD controller: i) the event duration signal (TResp) which represents the time spent from the detection instant of the current event to the current time instant, ii) $\Delta \mathrm{HR}$, iii) $\frac{\partial \Delta H R}{\partial t}$, iv) FSaO2 signal and v) $\frac{\partial F S a O 2}{\partial t}$. The coupled PD controller module synthesizes a signal, with an amplitude modulated by the controller's output, that will be used to drive the kinesthetic actuator. This modulated amplitude has a percentage range between $0 \%$ and $100 \%$, which is translated by the system to the specific input signal value to generate the equivalent acceleration delivered by the actuator (100\% amplitude (2V RMS) typically corresponds to a normalized acceleration of 13.7 $\mathrm{m} / \mathrm{s}^{2}$ ). In addition, the synthesized signal is delivered in a burst sequence of 4 bursts with a fixed stimulation duration of 3 seconds followed by a silent period of 2 seconds.

\subsection{Closed-loop algorithm}

Figure 1 shows a diagram of the closed-loop control algorithm. The loop is executed in real-time and all the variables are constantly calculated. The time passed since the beginning of the application is stored in variable $t$ while the event number for each detection is stored in $e(e=1,2, \ldots, N)$, where $\mathrm{N}$ is the total number of respiratory events during the whole night. The beginning, $t_{e}^{\text {start }}$ and end $t_{e}^{e n d}$ of each respiratory event $e$ are also available variables. When an event detection $e$ arrives, the amplitude of the stimulation burst at each time instant $(A(t, e))$ is obtained by the following equations:

$$
\begin{gathered}
A(t+1, e)=A(t, e)+a_{1} T R \operatorname{Resp}(t, e)+b_{1} \Delta H R(t, e) \\
+b_{2} \frac{\partial \Delta H R(t, e)}{\partial t}+c_{1}(100-F S a 02(t, e)) \\
+c_{2} \frac{\partial F S a 02(t, e)}{\partial(t)}
\end{gathered}
$$

where $a_{1}, b_{1}, b_{2}, c_{1}, c_{2}$ are the control coefficients, to be optimized.

\subsection{Preliminary evaluation}

A two-phases clinical study, approved by the ethics committee of the Grenoble University Hospital (EKINOX study) was designed to evaluate the proposed adaptive, closed-loop stimulation system. This work concerns the first phase of the study (titration phase), which was focused on the evaluation of the technical feasibility of the proposed closed-loop method as well as the definition of optimal control parameters.

12 patients were included in this first phase, from two centers in France: The University Hospitals of Grenoble and Tours. Patients were eligible for this acute study if they had a history of severe obstructive sleep apnea assessed by polysomnography (PSG) testing within the past 6 months (Apnea-hypopnea index (AHI) $>30$ episodes/h and $80 \%$ obstructive events). All patients underwent a full standard PSG which was used as a gold standard (Brainbox EEG-1042, Oxymeter Delta PTT II, software Coherence version 6.1.3.405, Braebon Ultima Airflow Pressure 


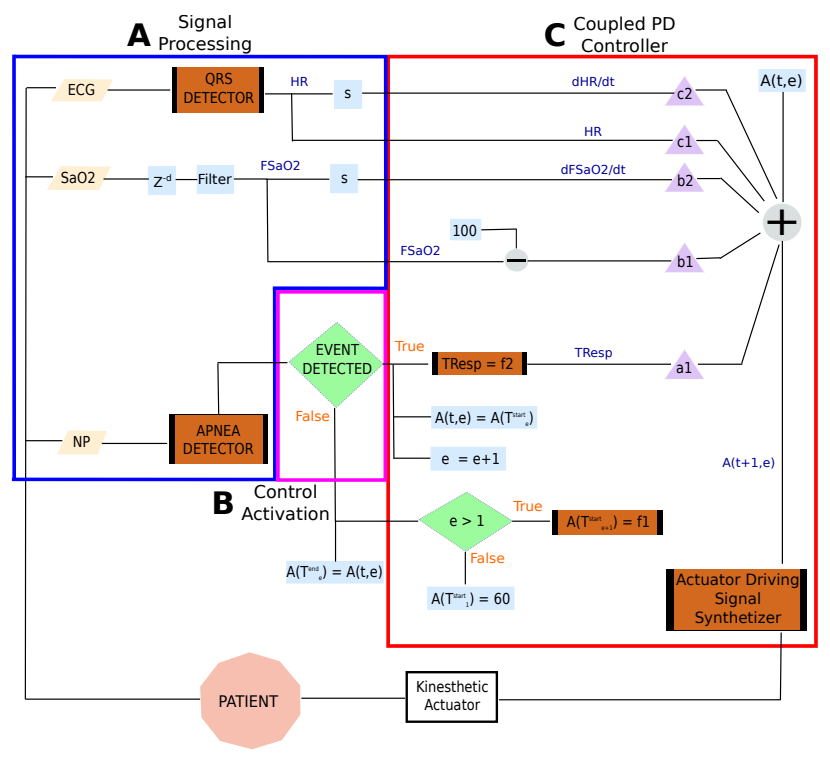

Figure 1. Diagram of the proposed closed-loop control. Signals presented as inputs to the control loop are: nasal pressure (NP), oxygen saturation $(\mathrm{SaO} 2)$ and electrocardiogram (ECG). ECG and NP signals pass through a QRS and an apnea detector, respectively, in order to determine the heart rate signal (HR) and the TResp signal which corresponds to the time passed into an apnea/hypopnea event. The control coefficients are represented as $a_{1}, b_{1}, b_{2}, c_{1}, c_{2}$. $T_{e}^{\text {start }}$ and $T_{e}^{\text {end }}$ are the beginning and the end of each detected respiratory event $e$. Finally, $A$ represents the amplitude value to be delivered by the controller.

Sensor, a surveillance camera and a computer). Simultaneously, NP, ECG and $\mathrm{SaO} 2$ signals were acquired and processed by the PASITHEA system. During this first phase, control coefficients were tested with borderline values, in order to estimate their impact on the controller.

A qualitative analysis was performed in order to characterize the patients response to the therapy. The analysis was based on the observation of respiratory event duration, $\mathrm{SaO} 2$ level and the cardiorespiratory response, along with a second analysis of the controller's behavior.

\section{Results}

This section will describe the physiological response of a representative patient to the proposed adaptive kinesthetic stimulation therapy. (Figure 2) shows the acquired data for a patients stimulated with the following control parameters: $\left(a_{1}=0.03, b_{1}=0, b_{2}=0, c_{1}=0.1, c_{2}=-2\right)$. The nasal pressure signal (Figure 2-A) is applied as input to the respiratory event detector. The output of the event detector is shown in Figure 2-B (red line). We observe in this example, the detection of a set of apnea events (marked with an "A"), and a set of hypopnea events ("H"). The stimulation signal, synthesized at the output of the controller and used to drive the kinesthetic actuator is shown in Figure 2-B, blue line. Note that the stimulation is only delivered when a respiratory event is detected and that the amplitude of the Stim signal changes over time, as a function of the physiological response of the patient. The number of bursts delivered depends on the event duration. In this case, the stimulation was able to stop all the respiratory events within the 6.1 seconds, and in most cases, with a limited number of bursts (one or two bursts). It is also important to highlight that the stimulation amplitude converges to a relatively low, stable value around $50 \%$, showing that the controller tends to minimize the delivered stimulation amplitude, while eliciting the desired physiological response. Another effect of the therapy is shown in figure 2 -C, where $\mathrm{SaO} 2$ signal remains around $95 \%$, showing no hypoxia events ( $\mathrm{Sa02}$ level below 90\%) due to the reduced duration of the respiratory episodes. Finally, figure 2-D shows the instantaneous heart rate (HR) signal, as detected from the ECG. It can be observed that there are no significant tachycardia events and the mean HR is low despite the number of respiratory events.

\section{Discussion}

Previous works have studied the possibility of using kinesthetic stimulation to treat sleep apnea, both in adults and infants. Although results from these studies suggest that this therapy may be useful, some limitations persist in order to be applicable in clinical practice. One of these limitations is related to the stimulation parameters that should be used and, in particular, to the stimulation amplitude. Indeed, if a very large stimulation amplitude is used, respiratory events may be stopped more easily but the patient's sleep can be significantly fragmented, while a too low amplitude will provoke no effect on respiratory events. An optimal stimulation amplitude should be defined between these extreme values.

In most previous works, stimulation parameters are constant for all patients and these parameters are defined heuristically from a test population. It seems obvious from previous results that each patient responds differently to a given set of kinesthetic stimulation parameters, implying the need of patient-specific stimulation parameter values. Moreover, changes in patient sleep stages and position during the night may have consequences on the optimal stimulation amplitude.

In this work, qualitative results showed that the controller is technically capable to adapt the stimulation and to converge to a minimum patient-specific amplitude, which is sufficient to elicit the desired physiological response. These results are encouraging concerning the feasibility of implementing this controller in therapy. However, further studies are necessary to evaluate the effect of the therapy, 


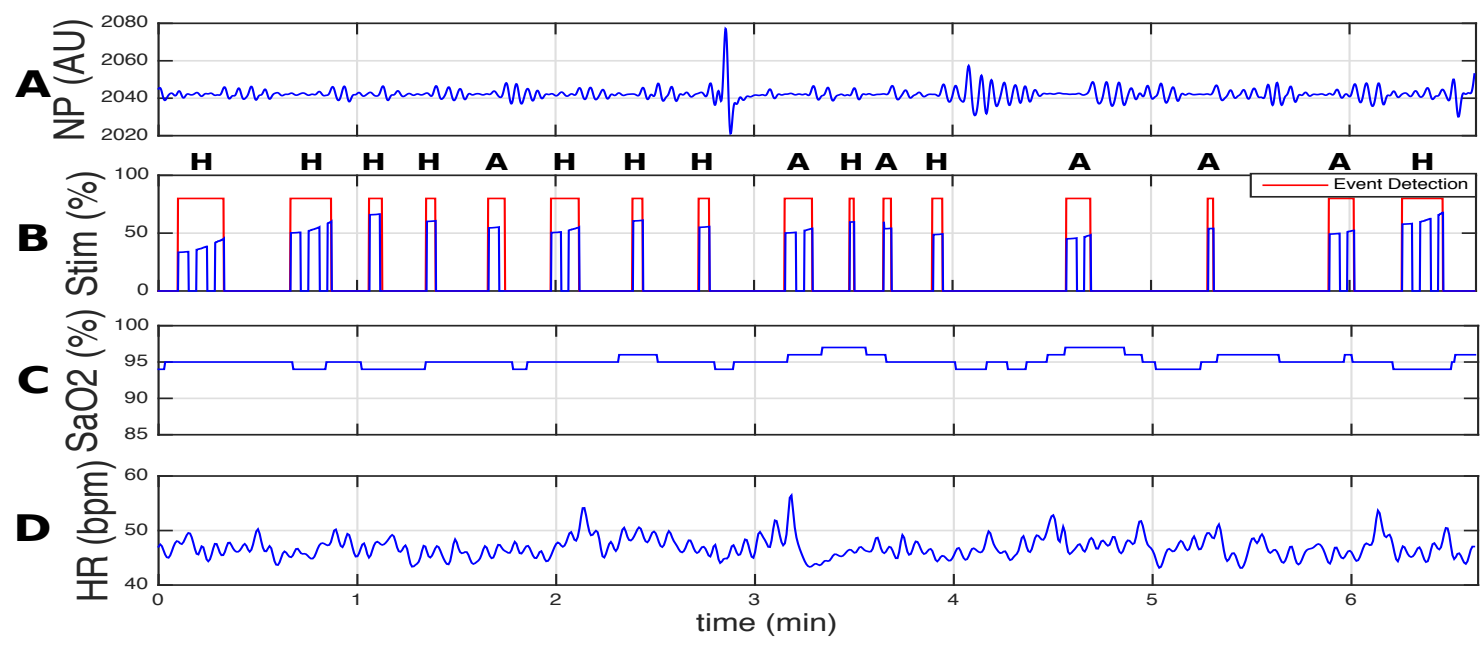

Figure 2. Cardiorespiratory response to adaptive kinesthetic stimulation. A) nasal pressure (NP), B) output of the respiratory event detector along with the amplitude signal of the kinesthetic stimulation (Stim), C) oxygen saturation (SaO2) and D) instantaneous heart rate (HR), as detected from the ECG.

performing a statistical comparison in the duration and frequency of the SAS events, as well as an analysis of the sleep structure of these patients. These analysis will be performed after the second phase of the EKINOx study. An important aspect that was observed during phase 1 is that the effectiveness of the therapy decreases as the night progresses. We hypothesize that the reason of this loss of effectiveness is due to a reduction of the mechanical coupling between the actuator and the patient's skin throughout the night.

\section{Conclusion}

This paper presented a novel control system for realtime detection and adaptive therapy delivery through kinesthetic stimulation, directed to patients suffering from SAS. Preliminary results of the effect of the adaptive stimulation shown in this work are very encouraging and they offer valuable information on the feasibility of the implementation of this controller in the therapy. Further works are directed to the clinical evaluation of this device.

\section{Ackbowledgment}

This project was supported by the French Research National Agency (ANR-12-TECS-0010 project).

\section{References}

[1] Jennum P, Riha RL. Epidemiology of sleep apnoea/hypopnoea syndrome and sleep-disordered breathing. European Respiratory Journal 2009;33(4):907-914.
[2] Stansbury RC, Strollo PJ. Clinical manifestations of sleep apnea. Journal of Thoracic Disease 2015;7(9):E298-E310.

[3] Somers VK, White DP, Amin R, Abraham WT, Costa F, Culebras A, Daniels S, Floras JS, Hunt CE, Olson LJ, et al. Sleep apnea and cardiovascular disease: An american heart association/american college of cardiology foundation scientific statement from the american heart association council for high blood pressure research professional education committee, council on clinical cardiology, stroke council, and council on cardiovascular nursing in collaboration with the national heart, lung, and blood institute national center on sleep disorders research (national institutes of health). Journal of the American College of Cardiology 2008;52(8):686717.

[4] Marin JM, Carrizo SJ, Vicente E, Agusti AG. Long-term cardiovascular outcomes in men with obstructive sleep apnoeahypopnoea with or without treatment with continuous positive airway pressure: an observational study. The Lancet 2005;365(9464):1046-1053.

[5] Kushida CA, Nichols DA, Holmes TH, Quan SF, Walsh JK, Gottlieb DJ, Simon Jr RD, Guilleminault C, White DP, Goodwin JL, et al. Effects of continuous positive airway pressure on neurocognitive function in obstructive sleep apnea patients: the apnea positive pressure long-term efficacy study (apples). SLEEP 2012;35(12):1593-1602.

[6] Hernández A, Guerrero G, Feuerstein D, Graindorge L, Perez D, Amblard A, Mabo P, Pépin JL, Senhadji L. Pasithea: An integrated monitoring and therapeutic system for sleep apnea syndromes based on adaptive kinesthetic stimulation. IRBM 2016;

[7] Feuerstein D, Graindorge L, Amblard A, Tatar A, Guerrero G, Christophle-Boulard S, Loiodice C, Hernandez AI, et al. Real- time detection of sleep breathing disorders. In Computing in Cardiology Conference (CinC), volume 42. 2015; 317-320. 